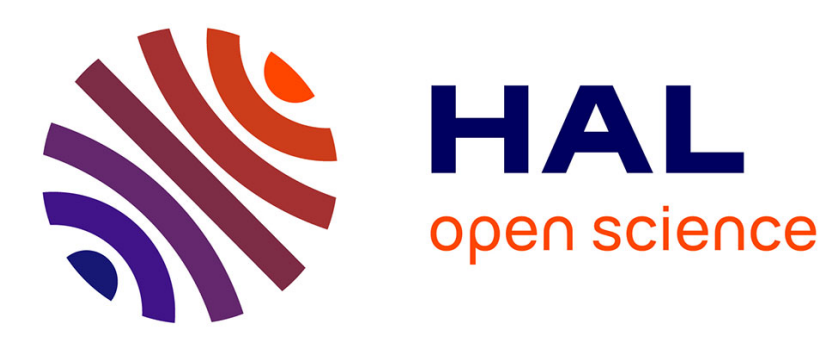

\title{
Walking Human Trajectory Models and Their Application to Humanoid Robot Locomotion
}

\author{
Isabelle Maroger, Olivier Stasse, Bruno Watier
}

\section{To cite this version:}

Isabelle Maroger, Olivier Stasse, Bruno Watier. Walking Human Trajectory Models and Their Application to Humanoid Robot Locomotion. IEEE/RSJ International Conference on Intelligent Robots and Systems (IROS), Oct 2020, Las Vegas, United States. 10.1109/IROS45743.2020.9341118 . hal02922573

\section{HAL Id: hal-02922573 \\ https://hal.laas.fr/hal-02922573}

Submitted on 28 Aug 2020

HAL is a multi-disciplinary open access archive for the deposit and dissemination of scientific research documents, whether they are published or not. The documents may come from teaching and research institutions in France or abroad, or from public or private research centers.
L'archive ouverte pluridisciplinaire HAL, est destinée au dépôt et à la diffusion de documents scientifiques de niveau recherche, publiés ou non, émanant des établissements d'enseignement et de recherche français ou étrangers, des laboratoires publics ou privés. 


\title{
Walking Human Trajectory Models and Their Application to Humanoid Robot Locomotion
}

\author{
I. Maroger, O. Stasse and B. Watier
}

\begin{abstract}
In order to fluidly perform complex tasks in collaboration with a human being, such as table handling, a humanoid robot has to recognize and adapt to human movements. To achieve such goals, a realistic model of the human locomotion that is computable on a robot is needed. In this paper, we focus on making a humanoid robot follow a human-like locomotion path. We mainly present two models of human walking which lead to compute an average trajectory of the body center of mass from which a twist in the 2D plane can be deduced. Then the velocities generated by both models are used by a walking pattern generator to drive a real TALOS robot [1]. To determine which of these models is the most realistic for a humanoid robot, we measure human walking paths with motion capture and compare them to the computed trajectories.
\end{abstract}

Index Terms-humanoid robot, human locomotion, path planning, benchmarking, clothoid, optimal control

\section{INTRODUCTION}

The context of this paper is human-humanoid interaction while walking. A classical approach consists in a robot passively following a human during an interaction [2]. However, a more efficient way is to use a model of the human walking behaviour. This allows the prediction and adaptation of the humanoid robot foot holds beforehand, in order to avoid the need to wait for a physical interaction. In the context of the French National Project Cobot, the targeted application is for a human and a humanoid robot to collaboratively hold a table. In this paper is proposed a study of the human gait to have a reference model for prediction which can be implemented in a humanoid robot Walking Pattern Generator (WPG).

\section{A. State of the art}

Human-humanoid interaction. In [3], Bussy et al. proposed a control scheme that allows a humanoid robot to perform a complex transportation scenario jointly with a human partner. The robot is guessing its human partner's intentions to proactively participate in the task. In a second phase, the robot (teleoperated by a human) takes over the leadership of the task to complete the scenario using motion primitives. The motion primitives are Stop, Walk, Side, Turn and Walk/Turn. They are pieced together using a finite state machine. The desired velocity used to drive the robot is inferred from this plan and by using a critically damped second order filter. To our knowledge, this is the first experiment where a humanoid robot is proactively generating a motion in collaboration with

This work is funded under the grant ANR-COBOT. LAAS-CNRS, Université de Toulouse, CNRS, UPS, France Toulouse, France

\{imaroger,ostasse,bwatier\}@laas.fr a human. With respect to this work, we are mostly focusing on the velocity model of the human behaviour, and checking its use on a human sized humanoid robot.

In [4] a controller is proposed to control two Nao humanoid robots in order to move collaboratively a table from one place to another. The controller stability is analyzed and proved to be stable. The system is able to lift a table-like object weighting more than half of the robot total weight. Unfortunately as we have only a very limited control over the human this work is not applicable in our context.

In [5] a human and a humanoid robot HRP-2 are walking and holding collaboratively a table while maintaining a ball on its top. This is carried out through visual servoing. An extension of this work has been presented in [6]. The HRP-4 robot is able to hold a bucket and a large box. The walking pattern generator used in this work is able to take as input a desired velocity. In our work, we propose a model of the human walk to take advantage of this walking pattern generator.

Humanoid robot walking pattern generator. Humanoid robot walk generation is a challenging topic. One common approach to address this problem is based on Zero Moment Point (ZMP) control, also named Center of Pressure (CoP) in biomechanics. Introduced in [7] and used in [8] and [9], the ZMP preview controller imposes to plan the robot footprints on the ground before the robot starts to walk which is not the most suitable for online application. Then, in [10] and [11] is introduced a WPG which only needs a velocity command to generate the ZMP and Body Center of Mass (shortened as $\mathrm{CoM}$ ) trajectories and the footprints. For this kind of WPG, only a velocity command on the CoM is needed. This command can be easily deduced from a smoothed reference CoM trajectory (i.e. without the perturbations induced by the steps). We will call this average trajectory: CoM path.

Following such dynamics is not simple as it depends on the mechanical capabilities of the robot, its stabilizer, its walking pattern generator and the used foot step planners as shown in [4]. We therefore choose to use the stabilizer and the walking pattern generator provided by PAL Robotics on the humanoid robot TALOS. Thanks to this reference velocity of the CoM it is possible to try to follow the model of human CoM velocity. This is different from [12], where the authors compute the ZMP trajectory and the foot positions from a generated CoM path using clothoid but do not follow the velocity model.

Multiples solutions exist to generate such a path, it can be as simple as following a straight line or more complex using B-spline curves [13], parametric curves like clothoids [12] or 


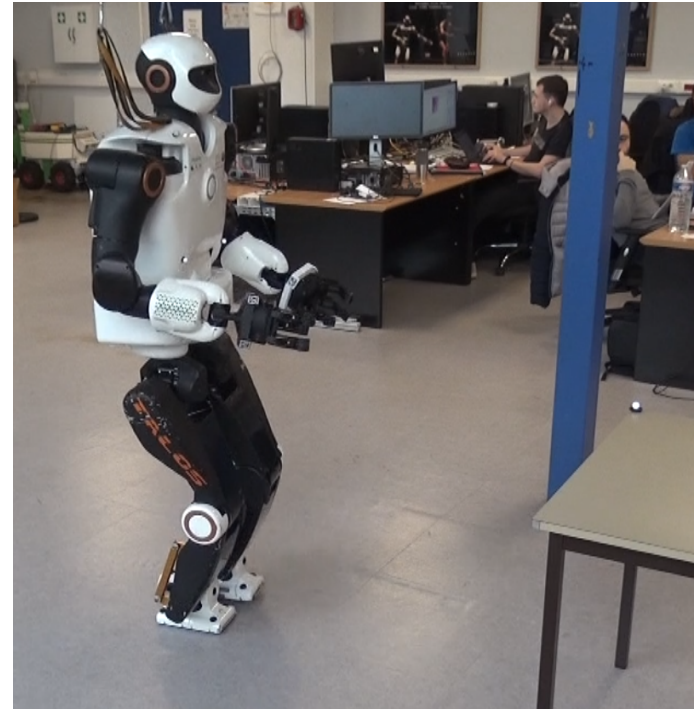

Fig. 1. TALOS robot produced by PAL Robotics walking toward a table

Optimal Control (OC) models like the unicycle model [14] or the model described in [15].

\section{B. Contribution}

In this article, the aim is to generate human-like CoM paths. To achieve this we generate trajectories using clothoids computation and an OC framework described in III. These trajectories are compared with humans CoM trajectories analyzed during biomechanics motion capture experiments performed on 10 subjects. Both models are recognized as good approximations of human CoM path during locomotion. The hypothesis is that the OC could provide better CoM paths which can be implemented on a TALOS humanoid robot (Fig. 11. For these experiments, a velocity command, computed from the CoM path, is given to the PAL Robotics WPG.

\section{HUMAN-LIKE TRAJECTORY GENERATION}

In this part are presented human locomotion models that define paths from a starting position with horizontal position $\left(x_{0}, y_{0}\right)$ and orientation $\theta_{0}$ to a goal position $\left(x_{f}, y_{f}\right)$ and orientation $\theta_{f}$ in a global predefined coordinate system. $\theta(t)$ is the instantaneous pelvis orientation during the locomotion phase. The problem is represented in Fig. 2. Two models have been implemented as part of this study.

\section{A. Nonholonomic locomotion model}

It has been shown that humans can frequently be approximated by nonholonomic system as they usually walk forward with the direction of their body tangent to their trajectory [16], [17]. Biologically, this can be explained by the anatomy of feet and legs. The nonholonomic locomotion problem, also known as the unicycle problem, is a well-known research topic in mobile wheeled robots [18]. However, when the goal is very close or if there is an obstacle on the way, humans decide to take sideward or oblique steps [15]. Moreover, the
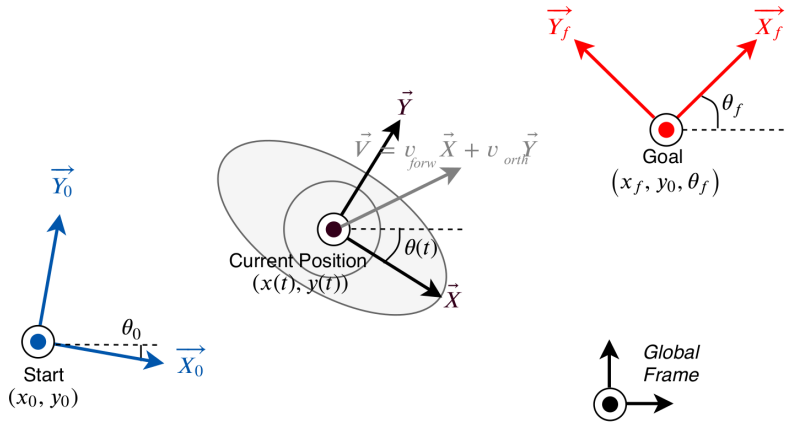

$\left(x_{f}, y_{0}, \theta_{f}\right)$

Fig. 2. Coordinate systems in the trajectory problem to solve
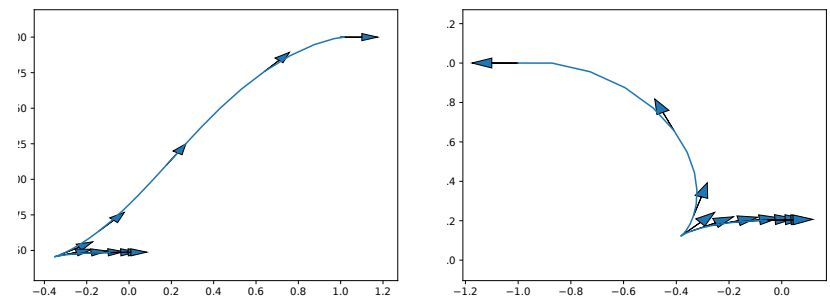

Fig. 3. Trajectories with a cusp generated with a python algorithm using OC based on Crocoddyl

unicycle model can generate trajectories with cusps, as Fig. 3 shows, far from a human-like behaviour.

\section{B. Clothoids}

Clothoid curves (also known as Cornu spirals) are frequently used for path-smoothing in robotics [14], [19], [20] and to mimic human CoM path during locomotion. Indeed, clothoid curves are interesting to generate trajectories because it has been demonstrated that clothoids arcs give good approximations of human trajectories during gait [21]. A clothoid satisfies the following system of ordinary differential equations:

$$
\left\{\begin{array}{l}
\dot{x}(s)=\cos \theta(s) \\
\dot{y}(s)=\sin \theta(s) \\
\dot{\theta}(s)=\kappa_{0}+\kappa_{1} s
\end{array}\right.
$$

with the following initial conditions : $x(0)=x_{0}, y(0)=y_{0}$ and $\theta(0)=\theta_{0} . \kappa_{0}$ is the initial curvature, $\kappa_{1}$ the sharpness of the curve and $s$ the curvilinear abscissa. From this system, the parametric expressions of a clothoid coordinates can be define as:

$$
\left\{\begin{array}{l}
x(s)=x_{0}+\int_{0}^{s} \cos \left(\theta_{0}+\kappa_{0} \xi+\frac{1}{2} \kappa_{1} \xi^{2}\right) d \xi \\
y(s)=y_{0}+\int_{0}^{s} \sin \left(\theta_{0}+\kappa_{0} \xi+\frac{1}{2} \kappa_{1} \xi^{2}\right) d \xi
\end{array}\right.
$$

Thus, one of the advantages of the clothoid curves is their linearly changing curvature which allows an easy control of the trajectory curvature. However, due to their transcendental nature, they cannot be solved analytically and the computational time needed to compute them remains important. In the robotics experiments using this model, we 


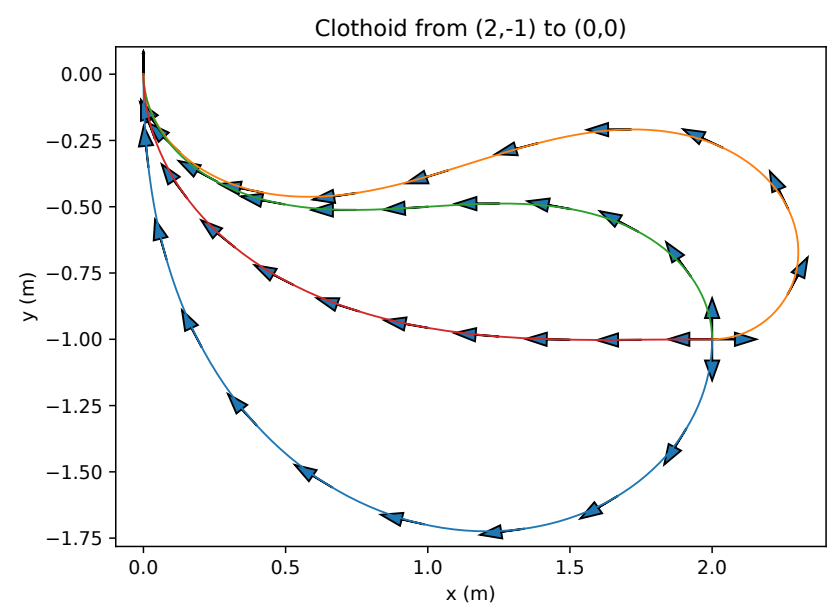

Fig. 4. Clothoids from $\left(2,-1,-\frac{\pi}{2}\right),(2,-1,0),\left(2,-1, \frac{\pi}{2}\right)$ and $(2,-1, \pi)$ to $\left(0,0, \frac{\pi}{2}\right)$ generated with a fitting algorithm (respectively in blue, orange, green and red)

suppose that the human behaviour follows $\mathrm{Eq} 1$

As the starting position and orientation of the robot and its goal position and orientation are known, a fitting method, described in [22], has been used to generate a clothoid arc between these two poses. The generated clothoid gives a CoM path from which the velocity command along the trajectory can be computed and given to the robot. The computed CoM velocity profile can then be transferred to the PAL Robotics WPG. Four trajectory examples are shown in Fig 4 . On this figure, the arrows represent the current orientation of the system during the gait.

\section{Human locomotion path model}

Reference [15] gives a realistic model of the human locomotion path with an OC model of the following form :

$$
\min _{X(.), U(.), T} \int_{0}^{T} \phi(X(t), U(t)) \mathrm{d} t
$$

under the following constraints :

$$
\begin{cases}\dot{X}=f(t, X(t), U(t)) & \text { Dynamical constraint } \\ X(0)=X_{0} & \text { Initial condition } \\ X(T)=X_{f} & \text { Final condition }\end{cases}
$$

where $X(t)$ and $U(t)$ are the state and control variables and $\phi(X(t), U(t))=\sum_{i=0}^{n-1} \alpha_{i} \phi_{i}(X(t), U(t))$ is an a priori unknown objective function with $n$ the number of base functions needed to describe the objective function.

The authors consider a holonomic locomotor system which

*All the source code can be found on https://github.com/ imaroger/walking_human_trajectory_models follows the following dynamics equation:

$$
\left\{\begin{array}{l}
\dot{x}=\cos \theta \cdot v_{\text {forw }}-\sin \theta . v_{\text {orth }} \\
\dot{y}=\sin \theta \cdot v_{\text {forw }}+\cos \theta \cdot v_{\text {orth }} \\
\dot{\theta}=\omega \\
\dot{v}_{\text {forw }}=u_{1} \\
\dot{v}_{\text {orth }}=u_{2} \\
\dot{\omega}=u_{3}
\end{array}\right.
$$

with $(x, y, \theta)$ the position and orientation of the locomotor system in the global frame and $\left(v_{\text {forw }}, v_{\text {orth }}, \omega\right)$ the forward, orthogonal and rotational velocities in the local frame as represented in Fig. 2. So in the OC problem expressed in eq. (3) and eq. (4), the state is $X=\left(x, y, \theta, v_{\text {forw }}, v_{\text {orth }}, \omega\right)^{T}$ and the control is $U=\left(u_{1}, u_{2}, u_{3}\right)^{T}$. In the robotics experiments using this model, we assume that the human behaviour follows Eq 5

Using inverse OC, the authors find out that the objective function that best fitted their measurements of human locomotion trajectories is :

$$
\begin{aligned}
\phi(X(t), U(t))=\alpha_{0}+\alpha_{1} u_{1}^{2}(t)+ & \alpha_{2} u_{2}^{2}(t)+\alpha_{3} u_{3}^{2}(t) \\
& +\alpha_{4} \psi\left(X(t), X_{f}\right)^{2}
\end{aligned}
$$

with $\psi\left(X(t), X_{f}\right)=\arctan \frac{y_{f}-y(t)}{x_{f}-x(t)}-\theta(t)$ and $\left(\alpha_{0}, \alpha_{1}, \alpha_{2}, \alpha_{3}, \alpha_{4}\right)=(1,1.2,1.7,0.7,5.2)$

In this paper, we adapt this OC problem in order to solve it with a Differential Dynamic Programming (DDP) solver [23] from the open-source Crocoddyl library [24]. For the interested reader more details on the DDP algorithm are given in [23]. The specifications of the implementation used here are given in [24]. This adaptation was a necessity as it seems that the MUSCOD software [25], which was used in [15], is discontinued.

In Crocoddyl, the dynamic of the system is given to the action model and the initial state is one of the parameter given to the shooting problem. However the DDP solver does not allow a strict final equality constraint. Thus, to satisfy the final constraint in Eq.(4), we need to add terms in the objective function to take into account the final state. This is called weak optimality and the final position may sometimes not exactly be reached at the end of the optimisation process. Eq. (6) becomes :

$$
\begin{gathered}
\phi(X(t), U(t))=\alpha_{0}+\alpha_{1} u_{1}^{2}(t)+\alpha_{2} u_{2}^{2}(t)+\alpha_{3} u_{3}^{2}(t) \\
+\alpha_{4} \psi\left(X(t), X_{f}\right)^{2}+\alpha_{5}\left(\left(x_{f}-x(t)\right)^{2}+\left(y_{f}-y(t)\right)^{2}\right) \\
+\alpha_{6}\left(\theta_{f}-\theta(t)\right)^{2}
\end{gathered}
$$

where the weights $\alpha_{5}=5$ and $\alpha_{6}=8$, have been heuristically found. Let us point out that we use the DDP solver already developed in the Crocoddyl library to solve our OC problem. To this end, we had to create a new action model in order to respect the dynamics of our system and the cost function we have chosen. 


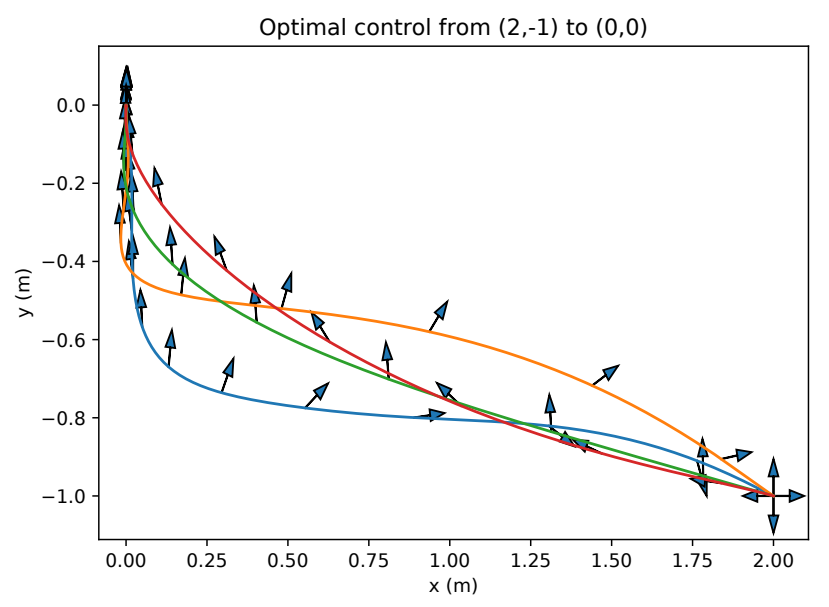

Fig. 5. Trajectories from $\left(2,-1,-\frac{\pi}{2}\right),(2,-1,0),\left(2,-1, \frac{\pi}{2}\right)$ and $(2,-1, \pi)$ to $\left(0,0, \frac{\pi}{2}\right)$ generated with a python algorithm using OC based on Crocoddyl (respectively in blue, orange, green and red)

In this study, as the duration time $T$ of the trajectory cannot be a free variable in the Crocoddyl solver, a similar problem is solved. Eq. (3) becomes :

$$
\min _{X(.), U(.)} \int_{0}^{T} \phi(X(t), U(t)) \mathrm{d} t
$$

Physically, $T$ represents the duration during which the locomotor system will perform the trajectory. As we want a realistic duration, we choose $T$ close to $\Delta t=$ $\frac{\sqrt{\left.\left(x_{f}-x_{0}\right)^{2}\right)+\left(y_{f}-y_{0}\right)^{2}}}{v}$ with $v$ the nominal speed of the system. As the path is necessarily longer that the straight line, the problem is solved for $T$ from $\Delta t$ to $\Delta t+200$ by increment of $5 \mathrm{~s}$. The $T$ which solves the problem and minimizes the objective function is kept. Thus, smoothed CoM trajectories can be computed along time and the velocity profiles can be deduced. Four trajectory examples obtained with this model are shown in Fig 5. The arrows represent the current orientation of the system during the gait.

\section{COMPARISON WITH HUMAN TRAJECTORIES}

Natural human behaviour during gait were measured and the obtained $\mathrm{CoM}$ trajectories were compared to the simulated CoM paths.

\section{A. Method}

Participants. Ten healthy subjects $(2$ females and 8 males) volunteered to take part in this experiment. Their ages ranged from 18 to 26 years old (average 23.3), their heights from $1.68 \mathrm{~m}$ to $1.84 \mathrm{~m}$ (average 1.77) and their masses from $58 \mathrm{~kg}$ to $105 \mathrm{~kg}$ (average 73.9). Each participant was informed of the experimental procedure and signed an informed consent form prior to the study. The study was conducted in accordance with the declaration of Helsinki and was approved by the University of Toulouse ethical committee. In order to preserve their natural behaviour, the participants were not familiar with this study and unaware

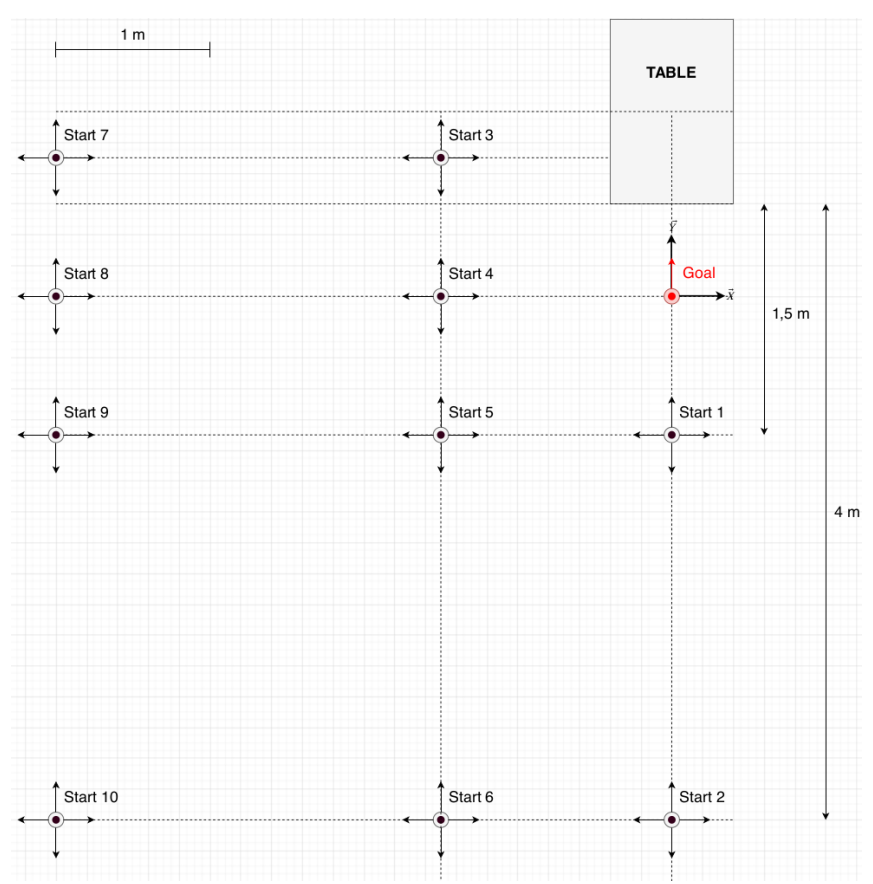

Fig. 6. Forty different starts and the goal position

of the expected results.

Experimental protocol. The subjects were asked to walk toward a table from 10 different starting positions with 4 different orientations at 90 degrees interval toward a goal position in front of the table (Fig. 6). For each starting position and each orientation, each volunteer performed at least three trials. Thus, each participant performed a total of 120 trials representative of common cases from 0.6 to $5.5 \mathrm{~m}$ away from the table.

Kinematics. Kinematics data were collected from passive markers recorded by fifteen infrared cameras (VICON, Oxford, United-Kingdom) sampled at $200 \mathrm{~Hz}$. The experimental setup is shown in Fig. 7) The CoM position during gait was approximated by the middle of the two postero-superior iliac spine [26]. All kinematics data were recorded in three dimensions.

Data reduction and analysis. Kinematics data were filtered using a 4th order, zero phase-shift, low-pass butterworth with a $10 \mathrm{~Hz}$ cutoff frequency. The CoM trajectory during each trial was then extracted in the horizontal plane and normalized from 1 to $100 \%$. For each starting position, a mean trajectory of the CoM was computed including all the subjects and at least one trial per condition. Then the simulated CoM trajectories were compared with the natural CoM trajectories of the subjects. 


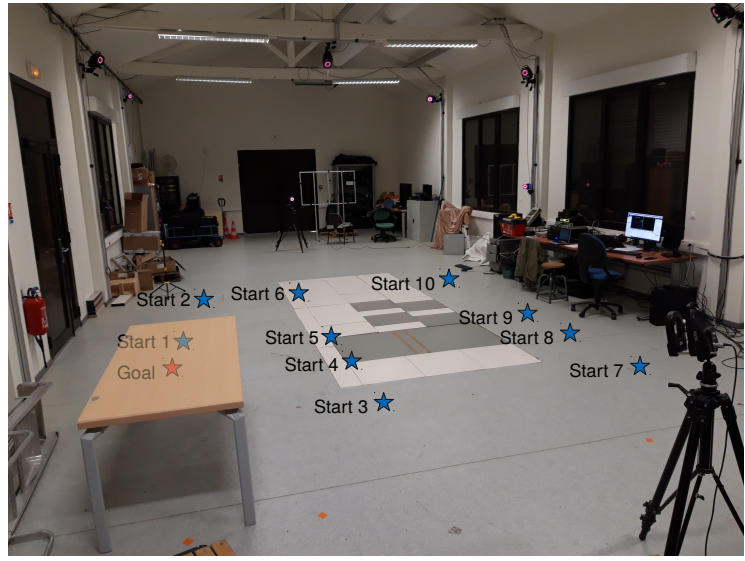

Fig. 7. Experimental setup to capture human trajectories

\section{B. Comparison}

Distance between the natural trajectories of humans and the simulated trajectories using clothoids and OC were then compared. For such analysis, a linear interpolation has been done to obtain the 2D coordinates of the CoM each percent along the path. Thus $N=100$ discrete positions are compared between the human CoM path and the simulated trajectories. The following average distance is defined :

$$
d=\frac{1}{N} \sum_{i=1}^{N} \sqrt{\left(x_{\text {real }}(i)-x_{\text {sim }}(i)\right)^{2}+\left(y_{\text {real }}(i)-y_{\text {sim }}(i)\right)^{2}}
$$

with $\left(x_{\text {real }}, y_{\text {real }}\right)$ the measurements and $\left(x_{\text {sim }}, y_{\text {sim }}\right)$ the generated data. In this study, we hypothesize that the best model corresponds to the lowest distance $d$ between the generated CoM paths and the mean subjects trajectories.

The mean trajectory for each starting position is computed as follows : $\left(\bar{x}_{j}, \bar{y}_{j}\right)=\frac{1}{10}\left(\sum_{i=1}^{10} x_{j}^{(i)}, \sum_{i=1}^{10} y_{j}^{(i)}\right)$ where $i$ represents the $i^{t h}$ subject and $j$ the position along the CoM path from 1 to $100 \%$. Thus, for each mean experimental starting position $\left(\bar{x}_{0}, \bar{y}_{0}\right)$, each orientation and each goal position $\left(\bar{x}_{100}, \bar{y}_{100}\right)$ the CoM path is computed using the clothoids and the OC models. The obtained trajectories for 3 different starting positions are represented on Fig 8 .

The distance $d$ between the experimental and the generated curves are then computed. Fig 9 compares 4 trajectories between the 2 models and the experimental path. For each of these examples, the distance $d$ is lower with the OC when compared to the clothoids.

\section{Results}

The computed distance $d$ between the 2 models and the mean experimental trajectories are represented in Fig 10 . These results show that the OC model is better than the clothoids. Indeed, the mean distance $d$ for all starting positions and all orientations is $0.186 \mathrm{~m}$ with the $\mathrm{OC}$ model and $0.606 \mathrm{~m}$ with the clothoids. This result is more
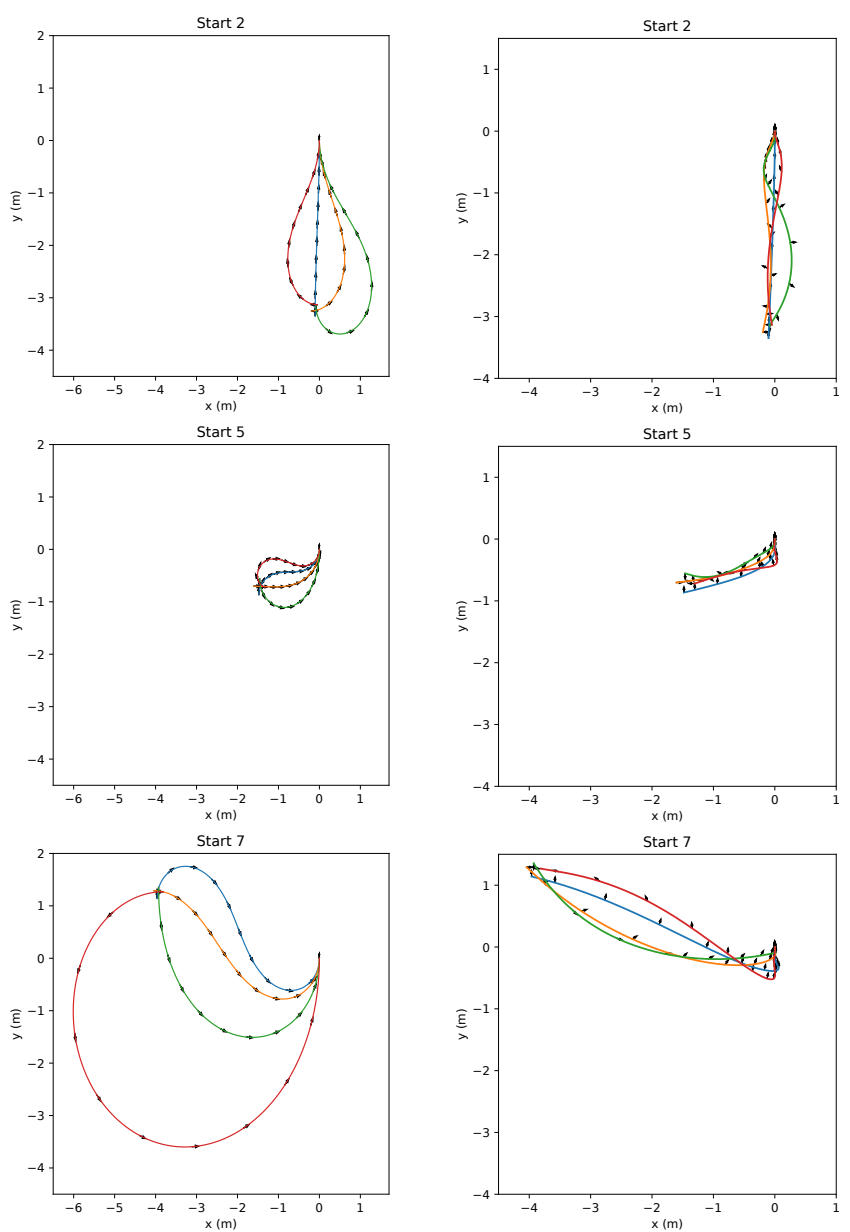

Fig. 8. Comparison between the generated trajectories (clothoid on the left and $\mathrm{OC}$ on the right) for 4 starting positions with the following starting orientations : $-\frac{\pi}{2} \mathrm{rad}$ in blue, $0 \mathrm{rad}$ in orange, $\frac{\pi}{2} \mathrm{rad}$ in green and $\pi \mathrm{rad}$ in red)

significant for $\theta_{0}=-\frac{\pi}{2}$ and $\theta_{0}=\pi$ where $d_{O C}=0.186 \mathrm{~m}$ and $d_{\text {Clothoids }}=0.939 \mathrm{~m}$.

Moreover, we can note that the farther the starting position is from the goal the greater the distance between the models and the measurements will be, especially for the model based on clothoids. In fact, the mean distances for all the starting position farther than $3 \mathrm{~m}$ are $d_{O C}=0.194 \mathrm{~m}$ and $d_{\text {Clothoids }}=0.796 \mathrm{~m}$.

\section{ApPliCATION ON TALOS ROBOT}

The aim of this work not only consists in determining the most human-like locomotion path but also in implementing this model on a TALOS robot which implies to respect some velocity and computation time limits.

\section{A. Experiment}

The main goal of this experiment is to make a TALOS robot walk in a human-like way toward a table. To achieve that, a motion capture system (20 infrared Qualisys Miqus M3 cameras sampling at $650 \mathrm{~Hz}$ with a $0.3 \mathrm{~mm}$ precision on the imaged area) is used to record in real time the 

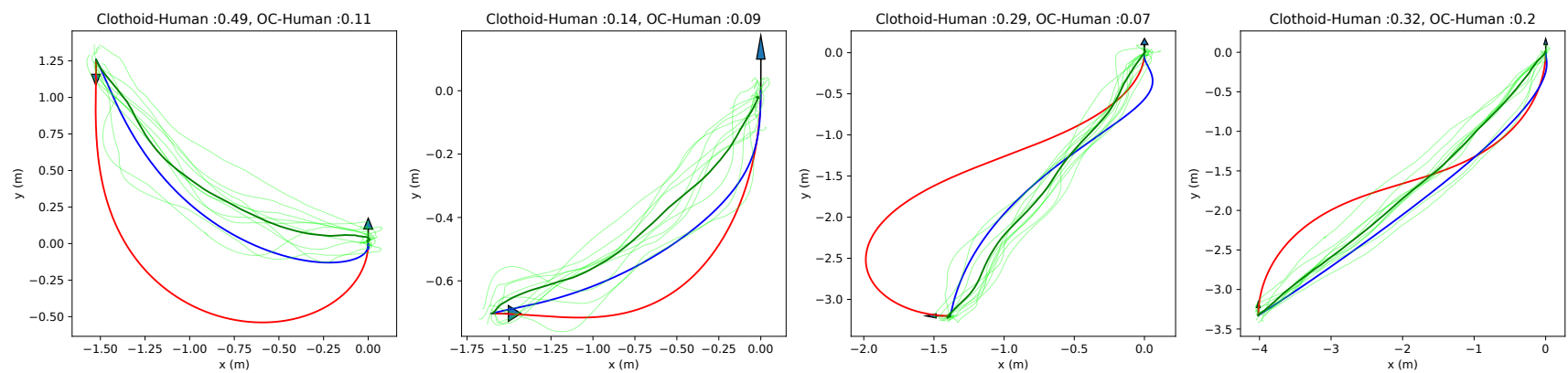

Fig. 9. Examples of comparison between the 2 presented models (clothoid in red and OC in blue) and measurements on 10 subjects (average trajectory in bold green and measured trajectories in light green) for start 3, 5, 6 and 10 respectively

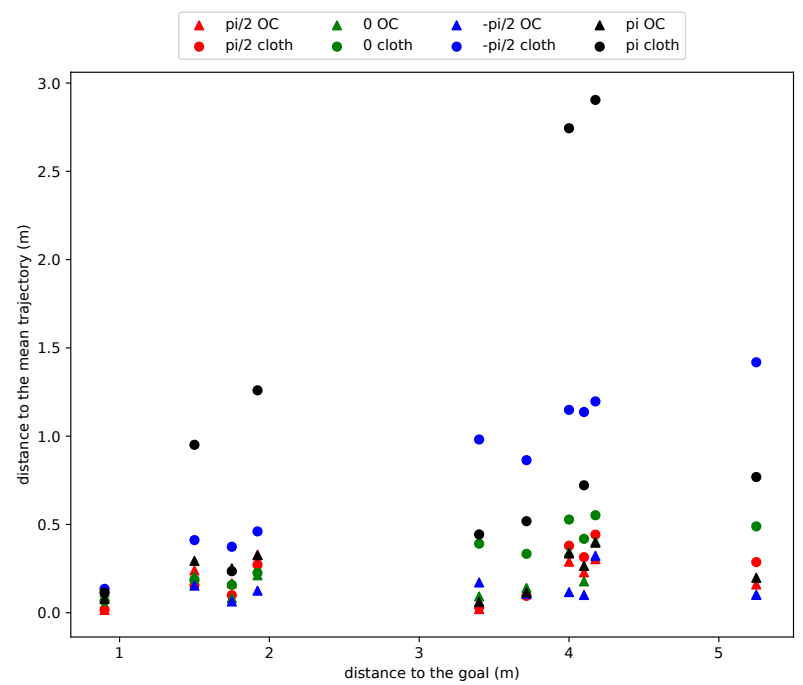

Fig. 10. Distance between the generated paths $(\bullet$ for clothoid and $\boldsymbol{\Lambda}$ for $\mathrm{OC})$ and the mean human trajectory as function of the distance from each starting position to the goal. Colors are related to the starting orientation in the global frame represented in Fig 6 for example to face the table the subject must have an orientation of $\theta_{f}=\frac{\pi}{2}$.

positions of the robot and the table. This allows to generate different trajectories using $\mathrm{OC}$ and clothoids and play them on a TALOS robot.

Both models generate a CoM path between the initial pose (where the robot initially is) and the final pose (at $90 \mathrm{~cm}$ from the closest edge of the table and oriented to face it). Then, a velocity command can be computed as follows:

$$
\left\{\begin{array}{l}
v_{x}(t)=\left(x_{\text {sim }}(t+1)-x_{\text {real }}(t)\right) \times f \\
v_{y}(t)=\left(y_{\text {sim }}(t+1)-y_{\text {real }}(t)\right) \times f \\
v_{\theta}(t)=\left(\theta_{\text {sim }}(t+1)-\theta_{\text {real }}(t)\right) \times f
\end{array}\right.
$$

with $\left(x_{\text {sim }}(t+1), y_{\text {sim }}(t+1), \theta_{\text {sim }}(t+1)\right)$ the next pose in the computed trajectory, $\left(x_{\text {real }}(t), y_{\text {real }}(t), \theta_{\text {real }}(t)\right)$ the current pose of the robot known thanks to the motion capture system and $f$ the sending rate. To be sent to the robot this velocity command must be expressed in the local frame of the robot. Then, this command can be given to the PAL Robotics WPG. In order to be performed safely by the robot it has to respect limits. No more than $0.1 \mathrm{~m} . \mathrm{s}^{-1}$ for the linear velocity along the $\vec{x}$ and $\vec{y}$ axis in the local coordinate system and no more than $7^{\circ} . s^{-1}$ for the angular velocity around the $\vec{z}$ axis.

To fulfill these requirements, a slowing parameter $\alpha$ has to be added to the clothoid description. Thus, Eq 1 becomes

$$
\left\{\begin{array}{l}
\dot{x}_{\alpha}(s)=\alpha \cos \theta(s) \\
\dot{y}_{\alpha}(s)=\alpha \sin \theta(s) \\
\dot{\theta}(s)=\kappa_{0}+\kappa_{1} s
\end{array}\right.
$$

with $\alpha \leq 1$, to linearly slow the system. To respect the linear velocity limit we take $\alpha=0.1$. With a $\mathrm{C}++$ algorithm that solves this system using the method presented in II-B, the computation duration is around $4.87 \cdot 10^{-4} \mathrm{~s}$ with a Intel ${ }^{\circledR}$ Core $^{\mathrm{TM}}$ i5-8400H CPU $2.50 \mathrm{GHz}$ processor. This allows to swiftly recompute the trajectory if the table is moved or if the robot gets away from its nominal trajectory. However, let us point out that there is a risk of collision against the table if the table is moved toward the robot because the velocity vector is always tangent to the trajectory when dealing with clothoid curve so the robot cannot walk backward.

The same idea is used to slow the trajectory generated with the $\mathrm{OC}, \mathrm{Eq} 5$ becomes :

$$
\left\{\begin{array}{l}
\dot{x}=\alpha \times\left(\cos \theta . v_{\text {forw }}-\sin \theta . v_{\text {orth }}\right) \\
\dot{y}=\alpha \times\left(\sin \theta \cdot v_{\text {forw }}+\cos \theta . v_{\text {orth }}\right) \\
\dot{\theta}=\omega \\
\dot{v}_{\text {forw }}=u_{1} \\
\dot{v}_{\text {orth }}=u_{2} \\
\dot{\omega}=u_{3}
\end{array}\right.
$$

with $\alpha \leq 1$, the smaller $\alpha$ is the greater $T$ will be. With a $\mathrm{C}++$ algorithm using Crocoddyl library as presented in II-C. the computation duration depends on $T$ and its optimisation can be expensive. So to be more precise with $\alpha=0.1$, it is around $37.1 \mathrm{~s}$ on the same processor. This is far too much to allow an online implementation in the robot. So to embed this trajectory model on the robot, the trajectory has to be previously generated and then played on the robot with no possibility to take into account the possible changes of environment, a change in the table location for example. 


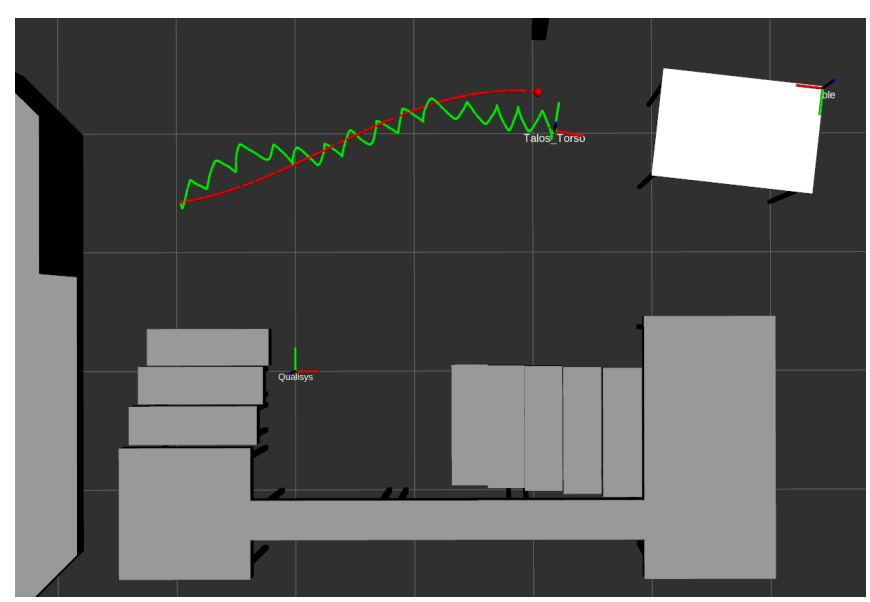

Fig. 11. Visualisation of the trajectory of the CoM of the robot (in green) during one experiment aiming to follow a clothoid trajectory (in red), in the upper right corner is the table (in white)

\section{B. Results}

Among 4 tests, the clothoid experiment produces the following results: the robot ends at about $0.065 \mathrm{~m}$ in $x$ and $0.205 \mathrm{~m}$ in $y$ from the goal position with an orientation of -0.028 rad from the goal orientation. Fig 11 shows a visualisation of one of this experiment on RViz. During one of the experiment, we moved the table and the robot successfully recomputed and followed a new clothoid in real time. One experiment with the robot following a clothoid curve is shown in the video which accompanies this paper.

Another conclusion which can be drawn from this experiment is that trying to make the robot follow a clothoid trajectory with the CoM velocity command imposed by the dynamic of the model does not result in the expected behaviour. Indeed, as we see in Fig.11, when the curvature increases the robot does not succeed in following the desired trajectory. Moreover, the rate at which the commands are sent seems crucial for the robot to perform the expected behaviour.

\section{Discussion}

In this paper, we study two a-priori human-like locomotion models. These models are compared to human trajectories in order to determine which one is the closest to human behaviour. Without contest, the OC model is closer than the model based on clothoid generation.

Human orientation and velocity analysis. In this work, two questions remains regarding the gait model. Which model gives the most human-like orientations of the pelvis during the gait? Are the velocities given by the models similar to human behaviour? First, as we see in Fig 4 and Fig,5, the clothoids generate a CoM velocity tangent to the orientation of the pelvis whereas in the OC model the CoM velocity can take various orientation not only in the antero-posterior direction but also along the medio-lateral axis. With the measures of the human trajectories, we could compute the pelvis orientation of the subjects during the gait. For ongoing works, we plan to analyse and compare this data to the generated orientation from the 2 models. This result could reinforce the validity of the OC model. Thus, it will be interesting to determine if the human orientation is tangent to its trajectory or not.

Furthermore, analysing the human velocities and comparing them to the hypothesized human-like velocities given by the models will be a necessity for further studies on the human behaviour during the gait.

Control over footsteps. We note that with no further control over the WPG parameters (like the stepping frequency, the duration of simple and double support), the robot cannot exactly follow a given dynamic. Therefore, if we can compute the footsteps and their timing along the trajectory, we could improve our results. This could be achieved using another WPG than the one currently provided by PAL Robotics. In future works, a more adaptable WPG should be developed. Moreover, analysis of the time, the amount of steps or the energy needed by the robot during locomotion will be considered in order to better assess its performance.

$O C$ cost function. In this paper, for the $\mathrm{OC}$ cost function we use the same weights as those proposed in [15]. Our measurements of human trajectories can be used with an inverse optimal control scheme to determine the optimal weights of the cost function described in Eq 7 These weights might be different from those of the cost function introduced in [15] as it was not possible to use the same solver. Instead we used a DDP solver. Moreover, the additional $\alpha_{5}$ and $\alpha_{6}$ weights have been heuristically determined. They could be optimized to provide more human-like results. Furthermore, to better match the problem stated in $\mathrm{Eq} 3$ and $\mathrm{Eq} 4$, the cost function defined in Eq 7 could be divided into a running cost function and a terminal cost function (Mayer additional term) which represents the final constraint. Future works will focus on this point.

Computational time of the OC model. A drawback of the OC approach is its computational cost compared to the clothoid. However, a faster computation time may be expected considering the formulation of the problem. First, the optimisation of $T$ which is partially responsible for the great computational cost of the model could be improved for further works. Then, we can try different approaches to solve the OC problem for example other software like the ACADO library [27]. Moreover, the DDP solver is providing gains to regulate the difference between the target trajectory and the current one. Investigating how this regulation could be used to correct the current robot state with respect to the target trajectory is one direction of improvement. 


\section{CONCLUSION}

In this paper, we compare two models of trajectory generation already used to describe human locomotion with the aim of finding the model which best fits human trajectories in order to help a humanoid robot follow and even predict human behaviour to improve their collaboration. The comparison of these models with measurements on 10 human subjects demonstrates that the trajectories generated with the model based on $\mathrm{OC}$ is much more human-like than the clothoid curves. Indeed, the distance between human trajectories and the generated paths is smaller for the OC than for the clothoid model for almost all of our 40 measured trajectories. However, the OC is computationally more time consuming which makes it hard to embed in a humanoid robot for real time application. The implementation of the models on a TALOS robot with the PAL Robotics stabilizer and WPG shows that the dynamic given by these models is hard to follow for the robot with only a control over the CoM velocity. Further works with an improved and faster OC model and an adaptable WPG are needed to achieve the targeted goal of using a model of the human behaviour within the WPG of a TALOS robot in order to ease its collaboration with humans.

\section{ACKNOWLEDGMENT}

The authors would like to thank the subjects that volunteered for the experiment as well as the reviewers, particularly for the advice on how to decrease the OC runtime for example with ACADO. This work was supported by the national research agency (ANR), through the project ANR-COBOT [18CE10-0003]. Study sponsor did not have a role in the study design, the data collection, analysis and interpretation, the writing of the manuscript and the decision to submit the manuscript for publication.

\section{REFERENCES}

[1] O. Stasse et al., "Talos: A new humanoid research platform targeted for industrial applications," in IEEE-RAS International Conference on Humanoid Robotics, 2017, pp. 689-695.

[2] K. Kosuge, H. Yoshida, and T. Fukuda, "Dynamic control for robothuman collaboration," in Proceedings of 1993 2nd IEEE International Workshop on Robot and Human Communication, 1993, pp. 398-401.

[3] A. Bussy, P. Gergondet, A. Kheddar, F. Keith, and A. Crosnier, "Proactive behavior of a humanoid robot in a haptic transportation task with a human partner," in IEEE International Workshop on Robot and Human Interactive Communication (ROMAN), 2012, pp. 962-967.

[4] L. Hawley and W. Suleiman, "Control framework for cooperative object transportation by two humanoid robots," 2019.

[5] D. J. Agravante, A. Cherubini, A. Bussy, P. Gergondet, and A. Kheddar, "Collaborative human-humanoid carrying using vision and haptic sensing," 2014, pp. 607-612.

[6] D. J. Agravante, A. Cherubini, A. Sherikov, P.-B. Wieber, and A. Kheddar, "Human-humanoid collaborative carrying," vol. 35, 2019, pp. 833-846.

[7] S. Kajita et al., "Biped walking pattern generation by using preview control of zero-moment point," in IEEE International Conference on Robotics and Automation, 2003.

[8] P.-B. Wieber, "Trajectory free linear model predictive control for stable walking in the presence of strong perturbations," in IEEE-RAS International Conference on Humanoid Robots, 2006.

[9] M. Morisawa et al., "Experimentation of humanoid walking allowing immediate modification of foot place based on analytical solution," in Proceedings 2007 IEEE International Conference on Robotics and Automation, 2007, pp. 3989-3994.
[10] A. Herdt, N. Perrin, and P. Wieber, "Walking without thinking about it," in 2010 IEEE/RSJ International Conference on Intelligent Robots and Systems, 2010, pp. 190-195.

[11] M. Naveau et al., "A Reactive Walking Pattern Generator Based on Nonlinear Model Predictive Control," in IEEE Robotics and Automation Letters, vol. 2, no. 1, 2017, pp. 10-17.

[12] M. Raković, S. Savić, J. Santos-Victor, M. Nikolić, and B. Borovac, "Human-inspired online path planning and biped walking realization in unknown environment," Frontiers in Neurorobotics, vol. 13, p. 36, 2019.

[13] M. Elbanhawi, M. Simic, and R. Jazar, "Continuous path smoothing for car-like robots using b-spline curves," vol. 80, 012015.

[14] S. Fleury, P. Soueres, J. . Laumond, and R. Chatila, "Primitives for smoothing mobile robot trajectories," IEEE Transactions on Robotics and Automation, vol. 11, no. 3, pp. 441-448, June 1995.

[15] K. Mombaur and J.-P. Laumond, "From human to humanoid locomotion-an inverse optimal control approach," Autonomous Robots, pp. 369-383, 2010.

[16] G. Arechavaleta, J. . Laumond, H. Hicheur, and A. Berthoz, "The nonholonomic nature of human locomotion: a modeling study," in The First IEEE/RAS-EMBS International Conference on Biomedical Robotics and Biomechatronics, 2006, pp. 158-163.

[17] G. Arechavaleta, J.-P. Laumond, H. Hicheur, and A. Berthoz, "On the nonholonomic nature of human locomotion," Autonomous Robots, vol. 25 , pp. 25-35, 2008.

[18] J.-P. Laumond, "Robot motionplanning and control," in Lectures Notes in Control and Information Sciences 229, Springer, 1998.

[19] Y. Kanayama and N. Miyake, "Trajectory generation for mobile robot," in Robotics Research: The Third International Symposium. Cambridge, Mass., 1986, p. 333-340.

[20] M. Brezak and I. Petrovic, "Path smoothing using clothoids for differential drive mobile robots," in IFAC Proceedings, 2011, pp. 1133-1138.

[21] G. Arechavaleta, J. Laumond, H. Hicheur, and A. Berthoz, "An optimality principle governing human walking," IEEE Transactions on Robotics, vol. 24, no. 1, pp. 5-14, Feb 2008.

[22] E. Bertolazzi and M. Frego, "G1 fitting with clothoids," Mathematical Methods in the Applied Sciences, vol. 38, 032014

[23] Y. Tassa, N. Mansard, and E. Todorov, "Control-limited differential dynamic programming," in IEEE International Conference on Robotics and Automation, 2014

[24] C. Mastalli et al., "Crocoddyl: An efficient and versatile framework for multi-contact optimal control," in IEEE International Conference on Robotics and Automation, 2020.

[25] H.G.Bock and K.J.Plitt, "A multiple shooting algorithm for direct solution of optimal control problems," in IFAC Proceedings Volumes, 1984, pp. 1603-1608.

[26] C. Gard, C. Miff, and A. Kuo, "Comparison of kinematic and kinetic methods for computing the vertical motion of the body center of mass during walking," in Human movement science, 2004, pp. 597-610.

[27] B. Houska, H. Ferreau, and M. Diehl, "ACADO Toolkit - An Open Source Framework for Automatic Control and Dynamic Optimization,' Optimal Control Applications and Methods, vol. 32, no. 3, pp. 298312, 2011. 